\title{
VIRTUALLY COMPACT SPECIAL HYPERBOLIC GROUPS ARE CONJUGACY SEPARABLE
}

\author{
ASHOT MINASYAN AND PAVEL ZALESSKII
}

\begin{abstract}
We prove that any word hyperbolic group which is virtually compact special (in the sense of Haglund and Wise) is conjugacy separable. As a consequence we deduce that all word hyperbolic Coxeter groups and many classical small cancellation groups are conjugacy separable.

To get the main result we establish a new criterion for showing that elements of prime order are conjugacy distinguished. This criterion is of independent interest; its proof is based on a combination of discrete and profinite (co)homology theories.
\end{abstract}

\section{INTRODUCTION}

One of the main themes of Geometric Group Theory is the study of groups which act on non-positively curved spaces. Two prominent classes of such groups is the class of hyperbolic groups (defined by Gromov in [13]) and the class of (virtually) special groups (introduced by Haglund and Wise in [16]). The intersection of these two classes is quite large and its elements, virtually special hyperbolic groups, have particularly nice properties.

Recall that a finitely generated group $G$ is said to be hyperbolic if its Cayley graph is a $\delta$-hyperbolic metric space, for some $\delta \geq 0$ (see, for example, [2]). On the other hand, $G$ is virtually compact special, if there is a finite index subgroup $H \leqslant G$, such that $H$ is isomorphic to the fundamental group of a compact special cube complex, whose hyperplanes satisfy certain combinatorial properties (see [16, Sec. 3]).

Since the original work of Haglund and Wise [16], many hyperbolic groups have been shown to be virtually special. For example, in the paper [15] Haglund and Wise showed that hyperbolic Coxeter groups are virtually compact special. In 34 Wise proved the same for finitely generated 1-relator groups with torsion, while in [1] Agol showed this for fundamental groups of closed hyperbolic 3-manifolds. In fact, Agol [1] proved that any hyperbolic group admitting a proper cocompact action on a CAT(0) cube complex is virtually compact special.

In this paper we study conjugacy separability of virtually compact special hyperbolic groups. Recall, that a group $G$ is conjugacy separable if for arbitrary non-conjugate elements $x, y \in G$ there is a homomorphism from $G$ to a finite group $F$ such that the images of $x$ and $y$ are not conjugate in $F$. Conjugacy separability can be regarded

Date: July 29, 2016.

2010 Mathematics Subject Classification. 20E26, $20 \mathrm{~F} 67$.

Key words and phrases. Conjugacy separable, virtually special groups, hyperbolic groups. 
as an algebraic analogue of solvability of the conjugacy problem in a group and has a number of applications. Most prominently it is used in proving residual finiteness of outer automorphism groups (see, for example, the discussion in [25, Sec. 2]).

Conjugacy separability is usually not easy to show, and, until recently, only a few classes of groups were known to satisfy it: virtually free groups [10], virtually surface groups [23] and virtually polycyclic groups [11, 29]. Note that in general conjugacy separability does not pass to finite index overgroups [12] or to finite index subgroups [24], therefore the adjective "virtually" is important.

A group $G$ is said to be hereditarily conjugacy separable if every finite index subgroup of $G$ is conjugacy separable. In [25] the first author showed that right angled Artin groups are hereditarily conjugacy separable. This result was subsequently used to prove conjugacy separability of Bianchi groups [7], 1-relator groups with torsion [26] and fundamental groups of compact 3-manifolds [17]. In fact, in [25] it was shown that any virtually compact special group $G$ contains a conjugacy separable subgroup of finite index. But it is still unclear whether such $G$ must necessarily be conjugacy separable itself. In the present paper we prove this in the case when $G$ is hyperbolic:

Theorem 1.1. Any virtually compact special hyperbolic group is hereditarily conjugacy separable.

Conjugacy separability of torsion-free virtually compact special hyperbolic groups was proved in [25, Cor. 9.11], so the actual novelty of Theorem 1.1 is in handling groups with torsion. In view of Agol's result [1, Thm. 1.1], the above theorem shows that every hyperbolic group, admitting a proper cocompact action on a CAT(0) cube complex, is hereditarily conjugacy separable. This gives an abundance of new examples of (hereditarily) conjugacy separable groups, some of which we mention in corollaries below.

For any Coxeter group $W$, Niblo and Reeves [27] constructed a cube complex $\mathcal{C}$ on which $W$ acts properly, and proved that the quotient complex $\mathcal{X}=W \backslash \mathcal{C}$ is compact if $W$ is hyperbolic. It follows that any hyperbolic Coxeter group is virtually compact special (originally this is due to Haglund and Wise [15]), hence we can use Theorem 1.1 to deduce:

Corollary 1.2. Any hyperbolic Coxeter group is hereditarily conjugacy separable.

Note that conjugacy separability of hyperbolic even Coxeter groups was proved in [6].

Another family of hyperbolic virtually compact special groups is given by groups with finite small cancellation presentations. Indeed, in [33] Wise proved that many classical small cancellation groups, including $C^{\prime}(1 / 6)$ and $C^{\prime}(1 / 4)-T(4)$ groups, act properly and cocompactly on CAT $(0)$ cube complexes. It is well-known that such groups are hyperbolic, so Agol's result [1, Thm. 1.1] applies and, together with Theorem 1.1, it yields

Corollary 1.3. Let $G$ be a group with a finite $C^{\prime}(1 / 6)$ or $C^{\prime}(1 / 4)-T(4)$ presentation. Then $G$ is hereditarily conjugacy separable.

Finally, Theorem 1.1 implies that any group acting properly and cocompactly on the hyperbolic 3-space is hereditarily conjugacy separable, because fundamental groups of closed hyperbolic 3-manifolds are virtually compact special by a combination of results of Bergeron and Wise [3] and Agol [1]. Thus we obtain the following statement: 
Corollary 1.4. Any uniform lattice in $P S L_{2}(\mathbb{C})$ is hereditarily conjugacy separable.

The above corollary could also be proved by combining results of Chagas and the second author [7, Thm. 2.5 or Thm. 2.7] with a different theorem of Agol from [1], claiming that closed hyperbolic 3-manifolds are virtually fibered.

Let us now say a few words about the proof of Theorem 1.1. One of the main difficulties in it is to separate conjugacy classes of torsion elements in a finite quotient. To this end we come up with a new approach (see Proposition 3.2) which employs (co)homological methods and is based on a result of K.S. Brown [5] allowing one to distinguish conjugacy classes of elements of prime order using group cohomology. In particular we obtain the following quite general result.

Theorem 1.5. Let $G$ be a residually finite group with $\operatorname{vcd}(G)<\infty$. If $G$ is cohomologically good then every element of prime order is conjugacy distinguished in $G$.

Recall that a residually finite group $G$ is cohomologically good, if the inclusion of $G$ in its profinite completion induces an isomorphism on cohomology with finite coefficients. An element $g \in G$ is said to be conjugacy distinguished if the conjugacy class $g^{G}$ is closed in the profinite topology on $G$ (thus $G$ is conjugacy separable if and only if each $g \in G$ is conjugacy distinguished). The claim of Theorem 1.5 can be restated by saying that two non-conjugate elements of prime order in $G$ are not conjugate in the profinite completion $\widehat{G}$; in other words, the embedding of $G$ in $\widehat{G}$ induces an injective map on the sets of conjugacy classes of elements of prime order in $G$ and in $\widehat{G}$. In Corollary 3.5 we prove that if, additionally, $G$ is finitely generated then this map is actually a bijection (in particular, every element of prime order in $\widehat{G}$ is conjugate to some element in $G$ ).

To prove Theorem 1.1 for a hyperbolic virtually compact special group $G$, we first show that $G$ is cohomologically good by proving that this property is stable under virtual retractions (Lemma 3.1), and combining this with some results from [16, 14, 20] (our argument actually does not make use of the hyperbolicity of $G$ and works, more generally, for almost virtual retracts of right angled Artin groups - see Proposition 3.8). It follows that Theorem 1.5 can be applied to separate the conjugacy classes of elements of prime order in $G$. After this we prove that every torsion element of $G$ is conjugacy distinguished essentially by induction on its order.

Acknowledgements. The authors would like to thank Marco Boggi, Frédéric Haglund, Dessislava Kochloukova, Ian Leary and Nansen Petrosyan for enlightening discussions. The second author was supported by Capes and CNPq.

\section{Preliminaries}

2.1. Notation. Given a group $G$, its subgroups $K, H$ and an element $g \in G$, we will write $C_{H}(g)=\left\{h \in H \mid h g h^{-1}=g\right\}$ to denote the centralizer of $g$ in $H$, and $N_{H}(K)=$ $\left\{h \in H \mid h K h^{-1}=K\right\}$ to denote the normalizer of $K$ in $H$.

2.2. Hyperbolic groups and quasiconvex subgroups. Recall that a geodesic metric space $Y$ is (Gromov) hyperbolic if there exists a constant $\delta \geq 0$ such that for any geodesic 
triangle $\Delta$ in $Y$, any side of $\Delta$ is contained in the closed $\delta$-neighborhood of the union of the other sides (cf. [2]). A subset $Z \subseteq Y$ is quasiconvex if there is $\varepsilon \geq 0$ such that for any two points $z_{1}, z_{2} \in Z$, any geodesic joining these points is contained in the closed $\varepsilon$-neighborhood of $Z$.

If $G$ is a group generated by a finite set $\mathcal{A} \subseteq G$, then $G$ is said to be hyperbolic if its Cayley graph $\Gamma(G, \mathcal{A})$ is a hyperbolic metric space. Similarly, a subset $S \subseteq G$ is quasiconvex if it is quasiconvex when considered as a subset of $\Gamma(G, \mathcal{A})$.

Quasiconvex subgroups are very important in the study of hyperbolic groups. Such subgroups are themselves hyperbolic and are quasi-isometrically embedded in $G$ (see [2]). Basic examples of quasiconvex subgroups in hyperbolic groups are centralizers of elements (see [4, Ch. III.Г, Prop. 3.9]); this fact will be important for our argument below.

2.3. Right angled Artin groups. A right angled Artin group is a group which can be given by a finite presentation, where the only defining relators are commutators of the generators. To construct such a group, one usually starts with a finite simplicial graph $\Gamma$ with vertex set $V$ and edge set $E$. One then defines the corresponding right angled Artin group $A=A(\Gamma)$ by the following presentation:

$$
A=\langle V \|[u, v]=1, \text { whenever }(u, v) \in E\rangle,
$$

where $[u, v]=u v u^{-1} v^{-1}$ is the commutator of $u$ and $v$.

For any subset $S \subseteq V$, the subgroup $A_{S}=\langle S\rangle \leqslant A$ is said to be a full subgroup of $A$. It is easy to see that $A_{S}$ is itself a right angled Artin group corresponding to the full subgraph $\Gamma_{S}$ of $\Gamma$, induced by the vertices from $S$. Moreover, $A_{S}$ is a retract of $A$ - see [25, Sec. 6].

Recall that a subgroup $H$, of a group $G$, is a virtual retract if $H$ is a retract of some finite index subgroup $K \leqslant G$. In other words, $H \subseteq K$ and there is a homomorphism $\rho: K \rightarrow H$ such that $\rho(K)=H$ and $\left.\rho\right|_{H}=\operatorname{id}_{H}$.

Let $\mathcal{V R}$ denote the class of all groups which are virtual retracts of finitely generated right angled Artin groups, and let $\mathcal{A} \mathcal{V} \mathcal{R}$ be the class consisting of all groups $G$ such that $G$ has a finite index subgroup from $\mathcal{V} \mathcal{R}$. We are interested in these specific classes of groups because of the following two results: in 16. Haglund and Wise proved that any virtually compact special group $G$ belongs to the class $\mathcal{A} \mathcal{V} \mathcal{R}$, and in [25] the first author showed that any group $H \in \mathcal{V} \mathcal{R}$ is hereditarily conjugacy separable.

2.4. Profinite topology. The profinite topology on a group $G$ is defined by taking finite index subgroups as a basis of neighborhoods of the identity element. This topology is Hausdorff, i.e., $\{1\}$ is a closed subset of $G$, if and only if the group $G$ is residually finite. In the latter case, $G$ embeds in its profinite completion, $\widehat{G}$, and the profinite topology on $G$ is precisely the restriction of the natural topology of $\widehat{G}$ to $G$.

A subset $S \subseteq G$ is said to be separable if it is closed in the profinite topology on $G$. Thus an element $x \in G$ is conjugacy distinguished if its conjugacy class $x^{G}=\left\{g x g^{-1} \mid g \in G\right\}$ is separable in $G$. It is not difficult to see that the latter is equivalent to the property that for any element $y \in G$, which is not conjugate to $x$, there is a finite group $F$ and a 
homomorphism $\phi: G \rightarrow F$, such that $\phi(y)$ is not conjugate to $\phi(x)$ in $F$. It follows that $G$ is conjugacy separable if and only if all of its elements are conjugacy distinguished.

2.5. Criteria for conjugacy separability. The next standard observation will be useful (cf. [24, Lemma 7.2]):

Lemma 2.1. Let $K$ be a subgroup of finite index in a group $G$ and let $x \in K$. If $x$ is conjugacy distinguished in $K$ then $x$ is conjugacy distinguished in $G$.

The following criterion was discovered by Chagas and the second author in [7]:

Proposition 2.2 ([7, Prop. 2.1]). Let $H$ be a normal subgroup of index $m \in \mathbb{N}$ in a group $G$ and let $x \in G$ be any element. Suppose that $H$ is hereditarily conjugacy separable and the centralizer $C_{G}\left(x^{m}\right)$, of $x^{m} \in H$, satisfies the following conditions:

(i) $x$ is conjugacy distinguished in $C_{G}\left(x^{m}\right)$;

(ii) each finite index subgroup of $C_{G}\left(x^{m}\right)$ is separable in $G$.

Then $x$ is conjugacy distinguished in $G$.

Note that the original condition (i) from [7, Prop. 2.1] required $C_{G}\left(x^{m}\right)$ to be conjugacy separable, however, it is easy to see that the proof (see also [6, Prop. 2.2] for an alternative argument) only uses the weaker assumption that $x$ is conjugacy distinguished in $C_{G}\left(x^{m}\right)$.

2.6. Profinite topology on virtually compact special groups. Let $\mathcal{V} \mathcal{S H}$ denote the class of all virtually compact special hyperbolic groups.

Remark 2.3. The class $\mathcal{V C S H}$ is closed under taking finite index subgroups and overgroups.

Indeed, it is immediate from the definitions that a finite index subgroup/overgroup of a virtually compact special group is still virtually compact special. On the other hand, it is well-known that a group is hyperbolic if and only if a finite index subgroup is hyperbolic (for instance, this follows from the fact that hyperbolicity is invariant under quasi-isometries - see [4, Ch. III.H, Thm. 1.9]).

The next statement easily follows from the work of Haglund and Wise in [16].

Lemma 2.4. Suppose that $G \in \mathcal{V C S H}$ and $g \in G$. Then

(a) the centralizer $C_{G}(g)$ also belongs to $\mathcal{V C S H}$;

(b) every finite index subgroup of $C_{G}(g)$ is separable in $G$.

Proof. Fix some finite generating set $\mathcal{A}$ of $G$. Since the group $G$ is hyperbolic, it is wellknown that centralizers of elements in $G$ are quasiconvex (see, for example, [4, Ch. III. $\Gamma$, Prop. 3.9]). Hence $C_{G}(g)$ is quasiconvex, so it is also hyperbolic (cf. [2, Lemma 3.8]). In [16. Cor. 7.8] Haglund and Wise proved that any quasiconvex subgroup of $G$ is virtually compact special, thus (a) is proved.

To prove (b), note that every finite index subgroup $N \leqslant C_{G}(g)$ is also quasiconvex (because there is a constant $c \geq 0$ such that every element of $C_{G}(g)$ is at distance no more 
than $c$ from an element of $N$ in the Cayley graph $\Gamma(G, \mathcal{A})$ ). Therefore $N$ is separable in $G$ by [16, Cor. 7.4 and Lemma 7.5].

Lemma 2.5. Any virtually compact special group $G$ has a finite index normal subgroup $H \triangleleft G$ such that $H \in \mathcal{V} \mathcal{R}, H$ is torsion-free and hereditarily conjugacy separable.

Proof. In [16] Haglund and Wise proved that every virtually compact special group $G$ has a finite index normal subgroup $H \triangleleft G$ such that $H \in \mathcal{V} \mathcal{R}$. Now, $H$ is torsion-free as right angled Artin groups are torsion-free, and $H$ is hereditarily conjugacy separable by [25, Cor. 2.1].

\section{Cohomological goodness and its applications to COnjugacy SEPARABILITY}

Recall that a group $G$ is cohomologically good, if the natural embedding $G \hookrightarrow \widehat{G}$, of the group in its profinite completion, induces an isomorphism on cohomology with finite coefficients. This notion was originally introduced by Serre in [30, Exercises in Sec. I.2.6].

Cohomological goodness of residually finite groups behaves nicely under certain free constructions and is stable under group commensurability (see [14, 20]). We begin this section with proving another useful permanence property:

Lemma 3.1. Suppose that $G$ is a residually finite cohomologically good group and $H$ is a virtual retract of $G$. Then $H$ is cohomologically good.

Proof. Since the cohomological goodness passes to subgroups of finite index (see [14, Lemma 3.2]), we may assume that $H$ is a retract of $G$. Let $f: G \rightarrow H$ be a retraction. Then the profinite topology on $G$ induces the full profinite topology on $H$ (see, for example, [28, Lemma 3.1.5]), hence the natural embedding $i: H \rightarrow G$ induces an injective continuous map $\widehat{i}: \widehat{H} \rightarrow \widehat{G}$ (cf. [28, Lemma 3.2.6]). Therefore, the functorial property of profinite completions shows that the retraction $f$ induces a retraction $\widehat{f}: \widehat{G} \rightarrow \widehat{H}$, giving rise to the following commutative diagram, where the vertical maps are the natural embeddings of the residually finite groups in their profinite completions:

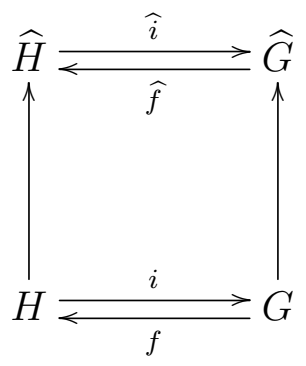

If $M$ is a finite $H$-module, we can turn it into a $G$-module by letting the kernel of $f$ act trivially on $M$. Then for any $n \in \mathbb{N} \cup\{0\}$, (1) induces the following commutative diagram of cohomology groups: 


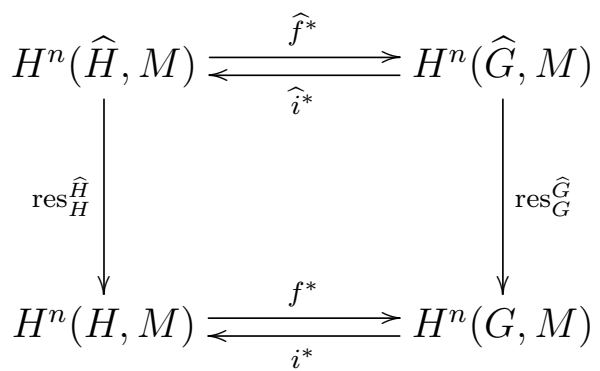

Since $f \circ i=\operatorname{id}_{H}$ and $\widehat{f} \circ \widehat{i}=\operatorname{id}_{\widehat{H}}$, we can deduce that $i^{*} \circ f^{*}$ and $\widehat{i}^{*} \circ \widehat{f}^{*}$ are identity maps on $H^{n}(H, M)$ and $H^{n}(\widehat{H}, M)$ respectively. In particular, the map $\widehat{f}^{*}$ is injective and the map $i^{*}$ is surjective.

Since $G$ is cohomologically good the right vertical arrow is a bijection and we need to show that so is the left vertical arrow. To see the injectivity, pick an element $h \in$ $H^{n}(\widehat{H}, M)$. Then $\left(f^{*} \circ \operatorname{res}_{H}^{\widehat{H}}\right)(h)=\left(\operatorname{res}_{G}^{\widehat{G}} \circ \widehat{f}^{*}\right)(h)$, implying that $h=0$ if $\operatorname{res}_{H}^{\widehat{H}}(h)=0$.

For surjectivity, observe that $i^{*} \circ \operatorname{res}_{G}^{\widehat{G}}=\operatorname{res}_{H}^{\widehat{H}} \circ \widehat{i}^{*}$ and the map on the left-hand side is surjective, hence $\operatorname{res}_{H}^{\widehat{H}}$ must also be surjective.

Thus $\operatorname{res}_{H}^{\widehat{H}}$ is an isomorphism, as required.

The next statement establishes a connection between cohomological goodness and separability of conjugacy classes of elements of prime order.

Proposition 3.2. Let $G$ be a residually finite cohomologically good group of finite virtual cohomological dimension. Suppose that $G$ splits as a semidirect product $G=H \rtimes\langle x\rangle$, where $H \triangleleft G$ is torsion-free and $x \in G$ has prime order $p$. Then the natural embedding of $G$ in $\widehat{G}$ induces an injective map between the conjugacy classes of finite subgroups in $G$ and in $\widehat{G}$.

Proof. Fix any integer $n>\operatorname{vcd}(G)$. Let $I$ [respectively, $\hat{I}$ ] denote the set of conjugacy classes of subgroups of order $p$ in $G$ [respectively, in $\widehat{G}$ ]. For every conjugacy class $\alpha \in I$ choose any subgroup $C_{\alpha}$, of order $p$, representing it in $G$. Since all elementary abelian $p$-subgroups of $G$ have rank at most 1 (as $G=H \rtimes\langle x\rangle$ and $H$ is torsion-free), we can apply a classical result of Brown (cf. Cor. 7.4 and the Remark below it in Ch. X of [5]), claiming that there is a canonical isomorphism

$$
\eta: H^{n}(G, \mathbb{Z} / p) \rightarrow \prod_{\alpha \in I} H^{n}\left(N_{G}\left(C_{\alpha}\right), \mathbb{Z} / p\right)
$$

Denote $N_{\alpha}=N_{G}\left(C_{\alpha}\right), \alpha \in I$. The above isomorphism $\eta$ can be defined as follows: for each $\alpha \in I$, the inclusion $N_{\alpha} \hookrightarrow G$ induces the restriction homomorphism $\operatorname{res}_{N_{\alpha}}^{G}$ : $H^{n}(G, \mathbb{Z} / p) \rightarrow H^{n}\left(N_{\alpha}, \mathbb{Z} / p\right)$, and $\eta=\prod_{\alpha \in I} \operatorname{res}_{N_{\alpha}}^{G}$ is the corresponding diagonal map.

For our purposes, it is actually more convenient to work with homology instead of cohomology. For each $\alpha \in I$, the inclusion $N_{\alpha} \hookrightarrow G$ induces the corestriction homomorphism 
$\operatorname{cor}_{N_{\alpha}}^{G}: H_{n}\left(N_{\alpha}, \mathbb{Z} / p\right) \rightarrow H_{n}(G, \mathbb{Z} / p)$. This gives a natural homomorphism

$$
\varphi: \bigoplus_{\alpha \in I} H_{n}\left(N_{\alpha}, \mathbb{Z} / p\right) \rightarrow H_{n}(G, \mathbb{Z} / p)
$$

defined by the property that the restriction of $\varphi$ to each direct summand $H_{n}\left(N_{\alpha}, \mathbb{Z} / p\right)$ is the $\operatorname{map} \operatorname{cor}_{N_{\alpha}}^{G}$.

Since $\mathbb{Z} / p$ is a field, the contravariant functor $\operatorname{Hom}_{\mathbb{Z} / p}(-, \mathbb{Z} / p)$ induces a natural isomorphism between $\operatorname{Hom}_{\mathbb{Z} / p}\left(H_{n}(F, \mathbb{Z} / p), \mathbb{Z} / p\right)$ and $H^{n}(F, \mathbb{Z} / p)$ for any group $F$ (for example by the Universal Coefficient Theorem, cf. [18, Sec. 3.1, pp. 196-197]). Applying this functor to (3) gives the map $\eta$ from (2).

If the map $\varphi$ was not injective then we would have a short exact sequence

$$
\{0\} \rightarrow K \rightarrow \bigoplus_{\alpha \in I} H_{n}\left(N_{\alpha}, \mathbb{Z} / p\right) \stackrel{\varphi}{\rightarrow} H_{n}(G, \mathbb{Z} / p) \rightarrow\{0\},
$$

where $K$ is a non-trivial vector space over $\mathbb{Z} / p$. Since $\mathbb{Z} / p$ is a field, the functor $\operatorname{Hom}_{\mathbb{Z} / p}(-, \mathbb{Z} / p)$ is exact, so it would give a short exact sequence

$$
\{0\} \rightarrow H^{n}(G, \mathbb{Z} / p) \stackrel{\eta}{\rightarrow} \prod_{\alpha \in I} H^{n}\left(N_{\alpha}, \mathbb{Z} / p\right) \rightarrow \operatorname{Hom}_{\mathbb{Z} / p}(K, \mathbb{Z} / p) \rightarrow\{0\} .
$$

The latter would contradict the fact that $\eta$ is surjective, as $\operatorname{Hom}_{\mathbb{Z} / p}(K, \mathbb{Z} / p) \neq\{0\}$. Therefore $\varphi$ is injective. A similar argument shows that $\varphi$ is also surjective, as $\eta$ is injective. Hence the homomorphism $\varphi$ in (3) is an isomorphism.

In particular, we see that if $\alpha_{1}$ and $\alpha_{2}$ are distinct elements of $I$ then

$$
\varphi\left(H_{n}\left(N_{\alpha_{1}}, \mathbb{Z} / p\right)\right) \cap \varphi\left(H_{n}\left(N_{\alpha_{2}}, \mathbb{Z} / p\right)\right)=\{0\} \text { in } H_{n}(G, \mathbb{Z} / p) .
$$

By the assumptions, for each $k=1,2, G=H \rtimes C_{\alpha_{k}}$, i.e., $G$ retracts onto $C_{\alpha_{k}}$. Therefore $N_{\alpha_{k}}$ also retracts onto $C_{\alpha_{k}}$, and hence the corestriction homomorphism $\operatorname{cor}_{C_{\alpha_{k}}}^{N_{\alpha_{k}}}$ : $H_{n}\left(C_{\alpha_{k}}, \mathbb{Z} / p\right) \rightarrow H_{n}\left(N_{\alpha_{k}}, \mathbb{Z} / p\right)$ is injective. Since $H_{n}\left(C_{\alpha_{k}}, \mathbb{Z} / p\right) \neq\{0\}$ for $k=1,2$ (as $\left.C_{\alpha_{k}} \cong \mathbb{Z} / p\right)$, (4) shows that the natural images of $H_{n}\left(C_{\alpha_{1}}, \mathbb{Z} / p\right)$ and $H_{n}\left(C_{\alpha_{2}}, \mathbb{Z} / p\right)$ in $H_{n}(G, \mathbb{Z} / p)$ must be distinct.

Now, arguing by contradiction, assume that there exist distinct $\alpha_{1}, \alpha_{2} \in I$ such that $C_{\alpha_{1}}$ is conjugate to $C_{\alpha_{2}}$ in $\widehat{G}$. We have the following commutative diagram coming from the natural inclusions:

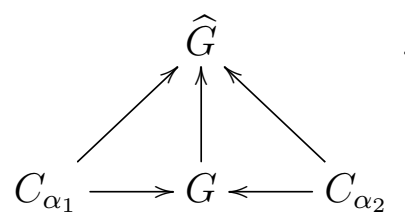

Since $C_{\alpha_{k}}$ is a closed subgroup of $\widehat{G}, k=1,2$, and $G$ is dense in $\widehat{G}$, this diagram induces the following commutative diagram of cohomology groups (for the vertical and diagonal arrows see [30, Sec. I.2.4 and Exercise 1) in Sec. I.2.6]): 
(6)

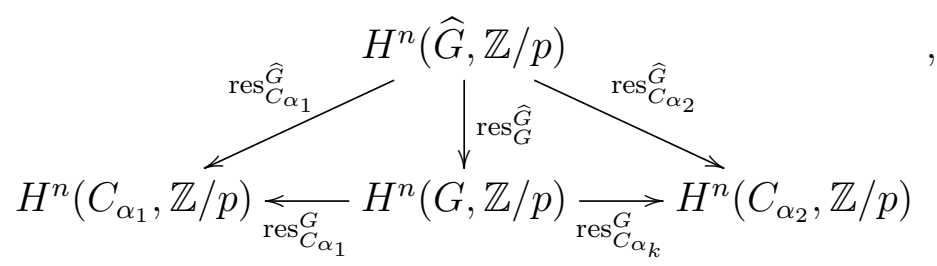

where $\operatorname{res}_{G}^{\widehat{G}}$ is an isomorphism by cohomological goodness of $G$.

Let us apply the $\operatorname{Hom}_{\mathbb{Z} / p}(-, \mathbb{Z} / p)$ functor to the diagram (6). Pontryagin duality between cohomology and homology of profinite groups (see [28, Prop. 6.3.6]) says that $\operatorname{Hom}_{\mathbb{Z} / p}\left(H^{n}(\widehat{G}, \mathbb{Z} / p), \mathbb{Z} / p\right)$ is naturally isomorphic to $H_{n}(\widehat{G}, \mathbb{Z} / p)$. On the other hand, for the discrete group $G, \operatorname{Hom}_{\mathbb{Z} / p}\left(H^{n}(G, \mathbb{Z} / p), \mathbb{Z} / p\right)$ may not be, in general, isomorphic to $H_{n}(G, \mathbb{Z} / p)$. However, since $\operatorname{Hom}_{\mathbb{Z} / p}\left(H_{n}(G, \mathbb{Z} / p), \mathbb{Z} / p\right) \cong H^{n}(G, \mathbb{Z} / p)$ (as observed above), the space $\operatorname{Hom}_{\mathbb{Z} / p}\left(H^{n}(G, \mathbb{Z} / p), \mathbb{Z} / p\right)$ can be thought of as the double dual of $H_{n}(G, \mathbb{Z} / p)$. Since there is always a canonical embedding of a vector space into its double dual, we obtain an injective homomorphism $\rho: H_{n}(G, \mathbb{Z} / p) \rightarrow H_{n}(\widehat{G}, \mathbb{Z} / p)$, which fits into the following commutative diagram:

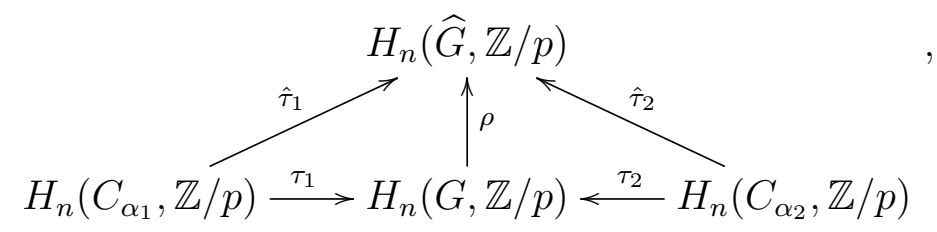

where $H_{n}(\widehat{G}, \mathbb{Z} / p)$ is the profinite homology of $\widehat{G}, \tau_{k}=\operatorname{cor}_{C_{\alpha_{k}}}^{G}$ and $\hat{\tau}_{k}=\operatorname{cor}_{C_{\alpha_{k}}}^{\widehat{G}}, k=1,2$.

By the assumption, there exists $g \in \widehat{G}$ such that $C_{\alpha_{2}}=g C_{\alpha_{1}} g^{-1}$. Hence we have

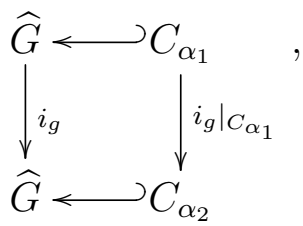

where $i_{g}: \widehat{G} \rightarrow \widehat{G}$ is the inner automorphism of $\widehat{G}$ given by $i_{g}(h)=g h g^{-1}$, for all $h \in \widehat{G}$, and $\left.i_{g}\right|_{C_{\alpha_{1}}}: C_{\alpha_{1}} \rightarrow C_{\alpha_{2}}$ is its restriction to $C_{\alpha_{1}}$. This leads to the following commutative diagram between the corresponding homology groups:

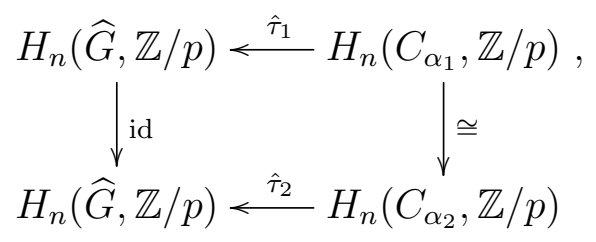

Note that the left vertical map is the identity on $H_{n}(\widehat{G}, \mathbb{Z} / p)$, as it is induced by an inner automorphism of $\widehat{G}$ (this is easy to prove directly, or one can use [30, Execise 1) in Sec. I.2.5] and apply the Pontryagin duality between $H^{n}$ and $H_{n}$ ). Therefore we can conclude that $\hat{\tau}_{1}\left(H_{n}\left(C_{\alpha_{1}}, \mathbb{Z} / p\right)\right)=\hat{\tau}_{2}\left(H_{n}\left(C_{\alpha_{2}}, \mathbb{Z} / p\right)\right)$ in $H_{n}(\widehat{G}, \mathbb{Z} / p)$. Thus, in view of 
injectivity of the map $\rho$ from $(7)$, in $H_{n}(G, \mathbb{Z} / p)$ we must have that $\tau_{1}\left(H_{n}\left(C_{\alpha_{1}}, \mathbb{Z} / p\right)\right)=$ $\tau_{2}\left(H_{n}\left(C_{\alpha_{2}}, \mathbb{Z} / p\right)\right)$. The latter gives a contradiction with the property that the natural images of $H_{n}\left(C_{\alpha_{1}}, \mathbb{Z} / p\right)$ and $H_{n}\left(C_{\alpha_{2}}, \mathbb{Z} / p\right)$ in $H_{n}(G, \mathbb{Z} / p)$ are distinct, which was proved above as a consequence of the fact that the map $\varphi$ in (3) is injective.

Therefore, $C_{\alpha_{1}}$ cannot be conjugate to $C_{\alpha_{2}}$ in $\widehat{G}$ if $\alpha_{1} \neq \alpha_{2}$ in $I$. This means that the inclusion $G \hookrightarrow \widehat{G}$ induces an injective map from $I$ to $\hat{I}$, as required.

We are now ready to prove Theorem 1.5 , stated in the Introduction.

Proof of Theorem 1.5. Let $p$ be a prime and let $x$ be an element of order $p$ in $G$. By the assumptions there exists a torsion-free normal subgroup $H \triangleleft G$, which has finite index in $G$. Denote $G_{1}=H\langle x\rangle \leqslant G$. Clearly $G_{1}$ has finite index in $G$, and $G_{1} \cong H \rtimes\langle x\rangle$. Therefore $G_{1}$ is residually finite and $\operatorname{vcd}\left(G_{1}\right)=\operatorname{vcd}(G)<\infty$. Moreover, $G_{1}$ is cohomologically good since this property passes to finite index subgroups and overgroups (see [14, Lemma 3.2]). Thus the group $G_{1}$ satisfies all the assumptions of Proposition 3.2 .

Consider any element $y \in G_{1}$, which is not conjugate to $x$. If $y$ and $x$ have different orders, then, using residual finiteness of $G_{1}$, we can find a finite quotient $M$, of $G_{1}$, where the images of $y$ and $x$ still have different orders, and hence they will not be conjugate in $M$. Therefore in this case $M$ will be a finite quotient of $G_{1}$ distinguishing the conjugacy classes of $y$ and $x$.

So, now we can suppose that $y$ also has order $p$. If $\langle y\rangle$ is not conjugate to $\langle x\rangle$ in $G_{1}$, then, by Proposition 3.2 , these subgroups are also not conjugate in $\widehat{G}_{1}$. Hence $y$ is not conjugate to $x$ in $\widehat{G}_{1}$, i.e., $y \notin x^{\widehat{G}_{1}}$. Now, the conjugacy class $x^{\widehat{G}_{1}}$ is closed in $\widehat{G}_{1}$, as $\widehat{G}_{1}$ is compact, so $x^{\widehat{G}_{1}} \cap G_{1}$ is a separable subset of $G_{1}$ which contains $x^{G_{1}}$ but avoids $y$. It follows that there is a finite quotient of $G_{1}$ distinguishing the conjugacy classes of $x$ and $y$.

Thus we can further assume that $\langle y\rangle$ is conjugate to $\langle x\rangle$ in $G_{1}$. Then $h y h^{-1}=z$ for some $h \in G_{1}$ and some $z \in\langle x\rangle$. Note that $z \neq x$ as $y$ is not conjugate to $x$ in $G_{1}$, by our assumption. Consequently, $z=\xi(z) \neq \xi(x)=x$, where $\xi: G_{1} \rightarrow\langle x\rangle$ is the natural retraction (coming from the semidirect product decomposition of $G_{1}$ ). Since the group $\langle x\rangle$ is abelian, we can conclude that $\xi(y)=\xi(z)$ is not conjugate to $\xi(x)$ in it, so $\langle x\rangle$ is a finite quotient of $G_{1}$ distinguishing the conjugacy classes of $x$ and $y$.

Thus we have considered all possibilities, showing that $x$ is conjugacy distinguished in $G_{1}$. It remains to apply Lemma 2.1 to conclude that $x$ is conjugacy distinguished in $G$, as required.

Proposition 3.2 shows that, under its assumptions, the natural inclusion $G \rightarrow \widehat{G}$ induces an injective map between the conjugacy classes of prime order subgroups in $G$ and in $\widehat{G}$. To complement this, we will now show this map is also surjective, provided $G$ has finitely many conjugacy classes of elements of prime order (the latter will be satisfied if $G$ is finitely generated - see Corollary 3.5 below).

Lemma 3.3. Suppose that $H$ is a cohomologically good group with $\operatorname{cd}(H)=n<\infty$. Then $\operatorname{cd}(\widehat{H}) \leq n$; in particular, $\widehat{H}$ is torsion-free. 
Proof. If $A$ is any simple discrete $\widehat{H}$-module, then $A$ is finite (because $\widehat{H}$ is compact and its action on $A$ is continuous $)$, so $H^{n+1}(\widehat{H}, A) \cong H^{n+1}(H, A)=\{0\}$ by cohomological goodness of $H$ and the assumption that $\operatorname{cd}(H)<n+1$. Hence $\operatorname{cd}_{p}(\widehat{H}) \leq n$ for every prime $p$ by [28, Prop. 7.1.4], therefore

$$
\operatorname{cd}(\widehat{H}):=\sup \left\{\operatorname{cd}_{p}(\widehat{H}) \mid p \text { prime }\right\} \leq n .
$$

Finally, since $\operatorname{cd}_{p}(C) \leq \operatorname{cd}_{p}(\widehat{H})<\infty$ for each prime $p$ and every closed subgroup $C \leqslant \widehat{H}$ (cf. [28, Thm. 7.3.1]), and $\operatorname{cd}_{p}(\mathbb{Z} / p)=\infty$ we can conclude that $\widehat{H}$ cannot contain subgroups of order $p$, for any prime $p$. Thus $\widehat{H}$ must be torsion-free, as claimed.

Proposition 3.4. Let $p$ be a prime and let $G$ be a residually finite cohomologically good group such that $\operatorname{vcd}(G)<\infty$ and $G$ contains finitely many conjugacy classes of subgroups (or, equivalently, elements) of order $p$. Then every element of order $p$ in the profinite completion $\widehat{G}$ is conjugate to some element of $G$.

Proof. Arguing by contradiction suppose that there is some element $\gamma \in \widehat{G}$, of order $p$, such that $C=\langle\gamma\rangle$ is not conjugate to any subgroup of $G$. By the assumptions, only finitely many conjugacy classes $\mathcal{C}_{1}, \ldots, \mathcal{C}_{k}$, of subgroups of order $p$ in $\widehat{G}$, intersect $G$ nontrivially. Since each $\mathcal{C}_{i}, i=1, \ldots, k$, is a compact subset of $\widehat{G}$, avoiding the finite subgroup $C$, there is a normal open subgroup $U$ of $\widehat{G}$ such that $C U \cap \mathcal{C}_{i}=\emptyset$ for every $i=1, \ldots, k$. Since $\operatorname{vcd}(G)<\infty, G$ contains a normal torsion-free subgroup $K$ of finite index. Then the closure $\bar{K}$, of $K$ in $\widehat{G}$, is naturally isomorphic to $\widehat{K}$, and hence it is torsion-free by Lemma 3.3 ( $K$ is cohomologically good by [28, Lemma 3.2.6] and $\operatorname{cd}(\mathrm{K})=\operatorname{vcd}(G)<\infty)$. So, after replacing $U$ by $U \cap \bar{K}$, we can assume that $U$ is torsion-free.

Now, $C U$ is an open subgroup of $\widehat{G}$, so $H=G \cap C U$ is a finite index subgroup of $G$, whose closure $\bar{H}$ in $\widehat{G}$ coincides with $C U$ (see [28, Prop. 3.2.2]). Since $H \cap \mathcal{C}_{i}=\emptyset$, $i=1, \ldots, k$, and every subgroup of order $p$ in $G$ is contained in some $\mathcal{C}_{i}$, we can conclude that $H$ has no elements of order $p$. On the other hand, since $C U$ is an extension of a torsion-free group $U$ by the cyclic group $C$, of order $p$, we see that $C U$ cannot contain non-trivial elements of finite orders other than $p$. Recalling that $H \leqslant C U$, allows us to conclude that $H$ is torsion-free.

Since $|G: H|<\infty$ we can argue as in the case of $K$ above (using Lemma 3.3) to deduce that $\bar{H}=C U$ must be torsion-free. The latter contradicts the fact that it contains $C$, completing the proof of the proposition.

Corollary 3.5. Suppose that $G$ is a finitely generated residually finite cohomologically good group with $\operatorname{vcd}(G)<\infty$. Then $G$ has finitely many conjugacy classes of subgroups of prime power order, and the natural inclusion of $G$ in $\widehat{G}$ induces a bijection between the conjugacy classes of elements (or subgroups) of prime order in $G$ and in $\widehat{G}$.

Proof. By the assumptions, $G$ has a normal torsion-free finite index subgroup $H$. It follows that there can be only finitely many primes $p$ such that $G$ contains some non-trivial $p$ subgroup. Let $p$ be such a prime. Since $G$ is cohomologically good, the same is true for $H$, so we can use a theorem of Weigel and the second author [32, Thm. B] claiming 
that $H^{n}(H, \mathbb{Z} / p)$ is finite for every $n \geq 0$. Since $\mathbb{Z} / p$ is a field, the Universal Coefficient Theorem tells us that the $\mathbb{Z} / p$-vector space $H^{n}(H, \mathbb{Z} / p)$ is the dual of $H_{n}(H, \mathbb{Z} / p)$, hence the latter is also finite. Therefore we can apply a result of Brown [5, Lemma IX.13.2] claiming that $G$ contains finitely many conjugacy classes of $p$-subgroups.

Thus we can use Proposition 3.4, to conclude that the natural map between the conjugacy classes of elements of prime order in $G$ and in $\widehat{G}$ is surjective. This map is injective by Theorem 1.5 , so the corollary is proved.

Remark 3.6. In the case when the group $G$ is virtually of type FP, Thm. 8.2 in the survey paper [19] asserts (without proof) that, with some extra work, a stronger version of Corollary 3.5 can be derived from a general result of Symonds [31, Thm. 1.1] (this was also confirmed to us by Symonds in a private communication).

An important tool for establishing cohomological goodness was discovered by Grunewald, Jaikin-Zapirain and the second author, and, independently, by Lorensen:

Proposition 3.7 ([14, Prop. 3.6],[20, Cor. 3.11]). Let $G=H *_{B=A^{t}}$ be an $H N N$-extension of a cohomologically good group $H$, where the associated subgroups $A$ and $B$ are also cohomologically good. Suppose that $G$ is residually finite, $H, A$ and $B$ are separable in $G$ and the profinite topology on $G$ induces the full profinite topologies on $H, A$, and $B$. Then $G$ is cohomologically good.

This allows us to show that in fact any group from the class $\mathcal{A V \mathcal { R }}$ is cohomologically good.

Proposition 3.8. Let $G \in \mathcal{A} \mathcal{V} \mathcal{R}$. Then $G$ is residually finite, cohomologically good and has finite virtual cohomological dimension.

Proof. By definition of the class $\mathcal{A} \mathcal{V} \mathcal{R}$, some finite index subgroup $H \leqslant G$ is a virtual retract of some right angled Artin group $A$. Right angled Artin groups are residually finite (see, for example, [9, Ch. 3, Thm 1.1]), hence $H$ and $G$ are both residually finite. The cohomological dimension $\operatorname{cd}(A)$, of $A$, is equal to the clique number of the associated graph (this follows from the fact that $A$ acts freely and cocompactly on a CAT(0) cube complex of the appropriate dimension - see [8, Sec. 3.6]), therefore $\operatorname{cd}(H) \leq \operatorname{cd}(A)<\infty$. Thus $\operatorname{vcd}(G)=\operatorname{cd}(H)<\infty$.

To show that $G$ is cohomologically good, we will first prove this for all right angled Artin groups (cf. [20, Thm. 3.15] and [21]). Let $B$ be a right angled Artin group corresponding to some finite simplicial graph $\Gamma$ with vertex set $V$. We will show that $B$ is cohomologically good by induction on $|V|$. If $|V|=0$ then $B=\{1\}$ and the claim holds trivially. Now, suppose that $|V|>0$ and choose any $S \subset V$ with $|V \backslash S|=1$. Then $B$ splits as an HNN-extension of $B_{S}$ over another full subgroup $B_{T}$, for some $T \subset S$ (see [25, Sec. 7]). Since $B_{S}$ and $B_{T}$ are a right angled Artin groups with less than $|V|$ generators, they are cohomologically good by the induction hypothesis. Recall that both $B_{T}$ and $B_{S}$ are retracts of $B$ and $B$ is residually finite, therefore these subgroups are separable in $B$ and the profinite topology of $B$ induces the full profinite topologies on these subgroups (cf. [28, Lemma 3.1.5]). Hence $B$ is cohomologically good by Proposition 3.7. 
Thus we have shown that any right angled Artin group is cohomologically good. Therefore, according to Lemma 3.1, the finite index subgroup $H \leqslant G$ is cohomologically good, as a virtual retract of $A$. Hence $G$ is itself cohomologically good by [14, Lemma 3.2].

Combining Theorem 1.5 with Proposition 3.8 and Lemma 2.5 we immediately obtain the following statement:

Corollary 3.9. Let $G$ be a virtually compact special group (or, more generally, let $G \in$ $\mathcal{A} \mathcal{V} \mathcal{R})$. Then every element of prime order is conjugacy distinguished in $G$.

\section{Proof of the MAIN RESUlT}

Before proving the main result we will need two more auxiliary statements.

Lemma 4.1. Let $G \in \mathcal{V C S H}$ and let $x \in G$ be an element of infinite order. Then $x$ is conjugacy distinguished in $G$.

Proof. By Lemma 2.5. $G$ has a normal subgroup $H$, of some finite index $m \in \mathbb{N}$, such that $H$ is hereditarily conjugacy separable. By the assumptions, $x^{m} \in H$ is an infinite order element in the hyperbolic group $G$, so its centralizer $C_{G}\left(x^{m}\right)$ is virtually cyclic (cf. [2, Prop. 3.5]). It follows that $C_{G}\left(x^{m}\right)$ is conjugacy separable. The second condition of Proposition 2.2 follows from Lemma 2.4.(b). Therefore we can use this proposition to conclude that $x$ is conjugacy distinguished in $G$, as required.

Corollary 4.2 (cf. [25, Cor. 9.11]). If $G \in \mathcal{V C S H}$ and $H \leqslant G$ is a torsion-free subgroup of finite index, then $H$ is hereditarily conjugacy separable.

Proof. Note that $H \in \mathcal{V C S H}$ by Remark 2.3, hence any element of infinite order is conjugacy distinguished in $H$ by Lemma 4.1. Since $H$ is torsion-free, the only element of finite order in $H$, the identity element, must also be conjugacy distinguished. Thus all elements of $H$ are conjugacy distinguished, i.e., $H$ is conjugacy separable.

Clearly the same argument applies to any finite index subgroup $K \leqslant H$. Therefore, $H$ is hereditarily conjugacy separable.

Proof of Theorem 1.1. Consider any group $G \in \mathcal{V C S H}$. Choose a torsion-free normal subgroup $H \triangleleft G$ such that $n=|G: H|$ is minimal (such $H$ exists by Lemma 2.5). We will prove the theorem by induction on $n$. If $n=1$ the statement holds because $H$ is hereditarily conjugacy separable by Corollary 4.2. So we can assume that $n>1$ and we have already established hereditary conjugacy separability for every group from $\mathcal{V} \mathcal{S H}$ which has a torsion-free normal subgroup of index less than $n$.

We will first show that $G$ is conjugacy separable. So, consider any element $x \in G$. If $x$ has infinite order, then $x$ is conjugacy distinguished in $G$ by Lemma 4.1. Thus we can suppose that $x$ has finite order.

Set $K=H\langle x\rangle$ and observe that $K \in \mathcal{V C S H}$ by Remark 2.3. If $|K: H|<n$ then $K$ is hereditarily conjugacy separable by the induction hypothesis, so $x$ is conjugacy distinguished in $K$. But then Lemma 2.1 implies that $x$ is conjugacy distinguished in $G$, as $|G: K| \leq|G: H|<\infty$. 
Therefore we can assume that $|K: H|=n=|G: H|$. It follows that $G=K$, i.e., $G=H\langle x\rangle \cong H \rtimes\langle x\rangle$, as $H$ is torsion-free and $x$ has finite order (which must then be equal to $n)$. We will now consider two cases.

Case 1: $n=p$ is a prime number. Then $x$ is conjugacy distinguished in $G$ by Corollary 3.9 .

Case 2: $n$ is a composite number. Thus $n=l m$ for some $l, m \in \mathbb{N}, 1<l, m<n$. We aim to use the criterion from Proposition 2.2, so let's check that all of its assumptions are satisfied.

Let $F=H\left\langle x^{m}\right\rangle \leqslant G$. Then $F \in \mathcal{V C S H}$ by Remark 2.3 and $F \cong H \rtimes(\mathbb{Z} / l)$. Thus $F$ is hereditarily conjugacy separable by the induction hypothesis, as $|F: H|=l<n$. Evidently, $F \triangleleft G$ and $|G: F|=m$. Every finite index subgroup of $C_{G}\left(x^{m}\right)$ is separable in $G$ by Lemma 2.4.(b), so it remains to check that $x$ is conjugacy distinguished in $C_{G}\left(x^{m}\right)$.

Set $H_{1}=C_{G}\left(x^{m}\right) \cap H$, and observe that $C_{G}\left(x^{m}\right)=H_{1}\langle x\rangle \cong H_{1} \rtimes(\mathbb{Z} / n)$. Moreover, in view of Remark 2.3, $H_{1} \in \mathcal{V C S} \mathcal{H}$ as $\left|C_{G}\left(x^{m}\right): H_{1}\right|=n<\infty$ and $C_{G}\left(x^{m}\right) \in \mathcal{V C S H}$ by Lemma 2.4.(a).

To verify that $x$ is conjugacy distinguished in $C_{G}\left(x^{m}\right)$, consider any element $y \in C_{G}\left(x^{m}\right)$ which is not conjugate to $x$ in $C_{G}\left(x^{m}\right)$. Since $x^{m}$ is central in $C_{G}\left(x^{m}\right)$, we can let $L$ be the quotient of $C_{G}\left(x^{m}\right)$ by $\left\langle x^{m}\right\rangle$, and let $\phi: C_{G}\left(x^{m}\right) \rightarrow L$ denote the natural epimorphism.

Clearly $\phi\left(H_{1}\right) \cong H_{1}$, as $H_{1} \cap \operatorname{ker} \phi=\{1\}$. Therefore $\phi\left(H_{1}\right)$ is torsion-free and $L=$ $\phi\left(H_{1}\right)\langle\phi(x)\rangle \cong H_{1} \rtimes(\mathbb{Z} / m)$, implying that $L \in \mathcal{V C S H}$ (by Remark 2.3). Consequently, $L$ is hereditarily conjugacy separable by the induction hypothesis, as $\left|L: H_{1}\right|=m<n$. Let us again consider two separate subcases.

Subcase 2.1: suppose that $\phi(x)$ and $\phi(y)$ are not conjugate in $L$. Then there is a finite group $M$ and a homomorphism $\psi: L \rightarrow M$ such that $\psi(\phi(x))$ is not conjugate to $\psi(\phi(y))$ in $M$. Thus the homomorphism $\eta=\psi \circ \phi: C_{G}\left(x^{m}\right) \rightarrow M$ will distinguish the conjugacy classes of $x$ and $y$, as required.

Subcase 2.2: assume that $\phi(x)$ is conjugate to $\phi(y)$ in $L$. Since $\operatorname{ker} \phi \subseteq\langle x\rangle$, we can deduce that there is $h \in C_{G}\left(x^{m}\right)$ such that $h y h^{-1}=z$, for some $z \in\langle x\rangle$.

Now, $z \neq x$, since we assumed that $y$ is not conjugate to $x$ in $C_{G}\left(x^{m}\right)$. Therefore $x=\xi(x) \neq \xi(z)=z$, where $\xi: C_{G}\left(x^{m}\right) \rightarrow\langle x\rangle$ is the natural retraction (coming from the decomposition of $C_{G}\left(x^{m}\right)$ as a semidirect product of $H_{1}$ and $\left.\langle x\rangle\right)$. Recalling that $\langle x\rangle$ is abelian, we see that $\xi(y)=\xi\left(h y h^{-1}\right)=\xi(z)$. Therefore $\xi(y)$ is not conjugate to $\xi(x)$ in the finite cyclic group $\langle x\rangle$. Thus we have distinguished the conjugacy classes of $x$ and $y$ in this finite quotient of $C_{G}\left(x^{m}\right)$.

Subcases 2.1 and 2.2 together imply that $x$ is conjugacy distinguished in $C_{G}\left(x^{m}\right)$. Therefore we have verified all of the assumptions of Proposition 2.2 (for $G$ and the finite index normal subgroup $F \triangleleft G$ ), so we can apply this proposition to deduce that $x$ is conjugacy distinguished in $G$. Thus Case 2 is completed.

Cases 1 and 2 exhaust all possibilities, so we have established conjugacy separability for any group $G \in \mathcal{V C S H}$, which possesses a torsion-free normal subgroup $H \triangleleft G$ of index $n$. If $K \leqslant G$ is any subgroup of finite index, then $K \in \mathcal{V C S} \mathcal{H}$ by Remark 2.3 and $H \cap K$ is a torsion-free normal subgroup in $K$ of index at most $n$. So, either using the induction 
hypothesis (if $|K:(H \cap K)|<n$ ) or the above argument (if $|K:(H \cap K)|=n$ ), we can conclude that $K$ is conjugacy separable as well. Hence $G$ is hereditarily conjugacy separable, and the step of induction has been established. This finishes the proof of the theorem.

\section{REFERENCES}

[1] I. Agol, The virtual Haken conjecture. Doc. Math., 18 (2013) 1045-1087. With an appendix by Agol, Daniel Groves, and Jason Manning.

[2] J. Alonso, T. Brady, D. Cooper, V. Ferlini, M. Lustig, M. Mihalik, M. Shapiro, H. Short, Notes on word hyperbolic groups. In: Short, H.B., ed. Group Theory from a Geometrical Viewpoint, Proc. ICTP Trieste, World Scientific Publishing Co., Inc., River Edge, NJ, pp. 3-63.

[3] N. Bergeron, D.T. Wise, A boundary criterion for cubulation. Amer. J. Math. 134 (2012), no. 3, 843-859.

[4] M. Bridson, A. Haefliger, Metric spaces of non-positive curvature. Grundlehren der Mathematischen Wissenschaften [Fundamental Principles of Mathematical Sciences], 319. Springer-Verlag, Berlin, 1999. xxii+643 pp.

[5] K.S. Brown. Cohomology of groups. Graduate Texts in Mathematics 87, Springer-Verlag, New YorkBerlin, 1982. $\mathrm{x}+306 \mathrm{pp}$.

[6] P.-E. Caprace, A. Minasyan, On conjugacy separability of some Coxeter groups and parabolicpreserving automorphisms. Illinois J. Math. 57 (2013), no. 2, pp. 499-523.

[7] S.C. Chagas, P.A. Zalesskii, Bianchi groups are conjugacy separable. J. Pure Appl. Algebra 214 (2010), no. 9, 1696-1700.

[8] R. Charney, An introduction to right-angled Artin groups. Geom. Dedicata 125 (2007), 141-158.

[9] C. Droms, Graph groups. PhD thesis, Syracuse University, 1983. Available from http://educ.jmu. edu/ dromscg/vita/thesis/

[10] J.L. Dyer, Separating conjugates in free-by-finite groups. J. London Math. Soc. (2) 20 (1979), no. 2, 215-221.

[11] E. Formanek, Conjugate separability in polycyclic groups. J. Algebra 42 (1976), no. 1, 1-10.

[12] A.V. Goryaga, Example of a finite extension of an FAC-group that is not an FAC-group (Russian). Sibirsk. Mat. Zh. 27 (1986), no. 3, 203-205, 225.

[13] M. Gromov, Hyperbolic groups. Essays in group theory, 75-263, Math. Sci. Res. Inst. Publ., 8, Springer, New York, 1987.

[14] F. Grunewald, A. Jaikin-Zapirain, P.A. Zalesskii, Cohomological goodness and the profinite completion of Bianchi groups. Duke Math. J. 144 (2008), no. 1, 53-72.

[15] F. Haglund, D.T. Wise, Coxeter groups are virtually special. Advances in Mathematics 224 (2010) 1890-1903.

[16] F. Haglund, D.T. Wise, Special cube complexes. Geom. Funct. Anal. 17 (2008), no. 5, 1551-1620.

[17] E. Hamilton, H. Wilton, P.A. Zalesskii, Separability of double cosets and conjugacy classes in 3manifold groups. J. Lond. Math. Soc. (2) 87 (2013), no. 1, 269-288.

[18] A. Hatcher, Algebraic topology. Cambridge University Press, Cambridge, 2002. xii+544 pp.

[19] P. Lochak, Results and conjectures in profinite Teichmüller theory. Galois-Teichmüller theory and arithmetic geometry, 263-335, Adv. Stud. Pure Math., 63, Math. Soc. Japan, Tokyo, 2012.

[20] K. Lorensen, Groups with the same cohomology as their profinite completions. J. Algebra 320 (2008), no. $4,1704-1722$.

[21] K. Lorensen, Corrigendum to "Groups with the same cohomology as their profinite completions" [J. Algebra 320 (2008) 1704-1722] J. Algebra 321 (2009), no. 2, 741.

[22] R.C. Lyndon, P.E. Schupp, Combinatorial group theory. Ergebnisse der Mathematik und ihrer Grenzgebiete, Band 89. Springer-Verlag, Berlin-New York, 1977. xiv+339 pp.

[23] A. Martino, A proof that all Seifert 3-manifold groups and all virtual surface groups are conjugacy separable. J. Algebra 313 (2007), no. 2, 773-781. 
[24] A. Martino, A. Minasyan, Conjugacy in normal subgroups of hyperbolic groups. Forum Math. 24 (2012), no. 5, 889-909.

[25] A. Minasyan, Hereditary conjugacy separability of right angled Artin groups and its applications. Groups Geom. Dyn. 6 (2012), no. 2, 335-388.

[26] A. Minasyan, P. Zalesskii, One-relator groups with torsion are conjugacy separable. J. Algebra 382 (2013), 39-45.

[27] G.A. Niblo, L.D. Reeves, Coxeter groups act on CAT(0) cube complexes. J. Group Theory 6 (2003), no. 3, 399-413.

[28] L. Ribes, P.A. Zalesskii, Profinite groups. Second edition. Ergebnisse der Mathematik und ihrer Grenzgebiete. 3. Folge. A Series of Modern Surveys in Mathematics [Results in Mathematics and Related Areas. 3rd Series. A Series of Modern Surveys in Mathematics], 40. Springer-Verlag, Berlin, 2010. xvi+464 pp.

[29] V.N. Remeslennikov, Conjugacy in polycyclic groups. (Russian) Algebra i Logika 8 (1969), 712-725.

[30] J.-P. Serre, Galois cohomology. Translated from the French by Patrick Ion and revised by the author. Springer-Verlag, Berlin, 1997. x+210 pp.

[31] P. Symonds, On cohomology isomorphisms of groups. J. Algebra 313 (2007), no. 2, 802-810.

[32] Th. Weigel and P.A. Zalesskii, Groups with infinite mod- $p$ Schur multiplier. J. Algebra 344 (2011), 70-77.

[33] D.T. Wise, Cubulating small cancellation groups. Geom. Funct. Anal. 14 (2004), no. 1, 150-214.

[34] D.T. Wise, The structure of groups with a quasiconvex hierarchy. Preprint (2011). Available from http://www.math.mcgill.ca/wise/papers.html.

(Ashot Minasyan) Mathematical Sciences, University of Southampton, Highfield, SoutHAMPTON, SO17 1BJ, United Kingdom.

E-mail address: aminasyan@gmail.com

(Pavel Zalesskii) Departamento de Matemática, Universidade de Brasília, 70910-900 BRASÍLIA-DF, BRAZIL.

E-mail address: pz@mat.unb.br 